\title{
Parapoxvirus orf virus infection induces an increase in interleukin-8, tumour necrosis factor-a, and decorin in goat skin fibroblast cells
}

\author{
Lingling Wang, Bingzhou Lu, Haixue Zheng, \\ Keshan Zhang, Xiangtao Liu \\ State Key Laboratory of Veterinary Etiological Biology, \\ Lanzhou Veterinary Research Institute of Chinese Academy of Agricultural Sciences, \\ Lanzhou, 730046, China \\ zks009@126.com
}

Received: February 23, 2016 Accepted: September 5, 2016

\begin{abstract}
Introduction: Orf virus (ORFV) is a prototype Parapoxvirus species in the Poxviridae family that causes serious zoonotic infectious disease. Goat skin fibroblast (GSF) cells are the major host targets of ORFV. Interleukin 8 (IL-8) and tumour necrosis factor (TNF)- $\alpha$ are known to play a vital role in immune response during viral infections. However, the manner of variation over time of their level of expression in GSF cells remains unclear. Material and Methods: In this study, quantitative enzyme-linked immunosorbent assay chips were used to detect changes in the levels of these cytokines expressed and secreted in GSF cells after ORFV infection. Results: Results showed that the expression of IL-8, TNF- $\alpha$, and decorin was upregulated in the cell lysates, and that secreted decorin and IL-8 were significantly increased in cell supernatant. Conclusion: The results provided possible approaches to elucidation of how ORFV infection initiates host cell immune response.
\end{abstract}

Keywords: goat skin fibroblasts, orf virus, cytokines.

\section{Introduction}

Orf virus (ORFV), a prototype species of the Parapoxvirus genus of the Poxviridae family, causes an infectious disease which naturally affects sheep and goats, and occasionally humans, cats, and some wild animals $(8,15)$. A recent study showed that ORFV can also be transmitted among humans (20). The infection in sheep and goats results in a debilitating mucocutaneous disease known as contagious ecthyma or orf which is characterised by proliferative lesions of the lips and muzzle, around the nostrils, and in the oral mucosa (14). ORFV infection is self-limited and generally lasts one to two months; however, it can be highly lethal when there is a mixed or secondary infection with other pathogens in lambs or kids (11). The disease has worldwide distribution and results in large economic losses.
ORFV has an envelope and a linear doublestranded DNA genome of approximately 134-139 kb long (4). The ORFV genome includes a large relatively conserved central core, which encodes transcription- and replication-related proteins, and inverted terminal repeats (ITRs) at each end, which encode virulence factors generally (27). These factors are mainly involved in modulating the immune response of the host. The orf virus interferonresistance gene (OVIFNR) encodes a protein resistant to type I interferon response (12). The vIL-10 gene production inhibits maturation of dendritic cells (DCs) and transcription of the $\mathrm{T}$ helper 1 (Th1) cell cytokines (16). Granulocyte-macrophage colonystimulating factor (GM-CSF) inhibitory factor (GIF) from ORFV suppresses functions of interleukin (IL)-2 and GM-CSF (3).

Cytokines play a vital role in immune response 
during viral infection, and some are implicated in ORFV infection. IL-8, also known as CXCL8, is a proinflammatory cytokine that is secreted at the infection site to recruit neutrophils and regulate pathological angiogenesis $(17,22)$. Tumour necrosis factor- $\alpha$ (TNF- $\alpha)$ is a multifunctional cytokine involved in regulating the processes of inflammation, immunity, apoptosis, and anti-tumour mechanism through its receptor (25). TNF- $\alpha$ influences the activation and migration of antigen-presenting cells (6). Decorin is a small leucine-rich proteoglycan that is involved in many pathological and physiological processes. It plays a key role in collagen fibrillogenesis (21), organisation of the extracellular matrix, activation of several tyrosine kinases (23), and leukocyte recruitment (24). It is interesting that ORFV infects the same host repeatedly although the host responds to the infection with a rapid immune and inflammatory reaction (10). Mechanisms by which ORFV elicits the host immune response might also influence cytokine expression.

Several studies have explored the consequence of cytokines being present in serum, blood, or immune cells after ORFV infection $(9,18)$. However, the changes in cytokine levels in their target cells in the early stages after infection have not been studied. Observation of these changes is critical to clarifying the signals through which ORFV-infected cells initiate the immune response. In this article, we detected the changes of several cytokines in the early stages after ORFV infection using a quantitative enzyme-linked immunosorbent assay (ELISA) chip.

\section{Material and Methods}

Cells and viral infection. Goat skin fibroblast (GSF) primary cells (purchased from the Kunming Cell Bank, Chinese Academy of Sciences) were grown in Dulbecco's modified Eagle's medium (DMEM) containing 10\% foetal bovine serum (FBS) at $37^{\circ} \mathrm{C}$ with $5.0 \% \quad \mathrm{CO}_{2}$. ORFV LY/2012/China strain (isolated and stored by State Key Laboratory of Veterinary Etiological Biology, China) was propagated in GSF cells, and the cell-free supernatant of the cell culture was used for the experiment. For infection, cell monolayers of GSF cells were grown in six-well plates until $80 \%$ confluence was reached. The cells were then infected with ORFV $\left(2.0 \times 10^{6} \mathrm{TCID}_{50}\right.$ per well $)$ in DMEM without FBS and incubated at $37^{\circ} \mathrm{C}$ for $1 \mathrm{~h}$, after which the liquid was replaced by fresh DMEM with $2 \%$ FBS. Equal volumes of phosphate-buffered saline (PBS) were added to the culture and the control well. An Olympus CKX41 microscope was used to observe the morphology of GSF cells infected with ORFV.
Sample collection. The GSF cells and supernatants were collected at $0.5,1,2,4,8$, and $12 \mathrm{~h}$ after ORFV infection. The cell-free supernatants were obtained by centrifugation at $2000 \mathrm{~g}$ for $5 \mathrm{~min}$. The cell lysates were washed three times with phosphate buffer and then prepared with lysis buffer (RayBiotech, USA) with $1 \times$ protease inhibitor cocktail (Thermo Scientific, USA). Lysates were homogenised twice by vortex and stored at $-80^{\circ} \mathrm{C}$ before use in the experiment.

Cytokine quantification. Several cytokines in the cell lysates and supernatants were detected using Ovine Cytokine Array 1 (cat. no. QAO-CYT-1, RayBiotech, USA) according to the manufacturer's instructions. The samples and diluted standard protein were added to the wells of a glass slide and incubated overnight at $4^{\circ} \mathrm{C}$ for better reaction of the antibody array chip and samples. Biotinylated secondary antibodies (which functioned as detectable antibodies) were then added and successively incubated after decanting the liquid from the wells and washing five times with wash buffers I and II included in the kit. Cy3 equivalent dye-streptavidin was added to combine with the biotin label of the secondary antibodies. The concentration of cytokines was measured as the mean fluorescence intensity of the streptavidin-bkg signal. Signals were detected using the GenePix 4000B Microarray Scanner (Molecular Devices, USA) through Cy3 channel (wavelength - $532 \mathrm{~nm}$, resolution - $42 \mathrm{um}$ ). Standard curves were generated from the serial dilutions of the cytokine standards.

\section{Results}

The GSF cell morphological changes are shown in Fig. 1. The concentrations of the samples were calculated by the standard curves. IL- 8 , TNF- $\alpha$, and decorin were detectable in the samples and their concentrations in the cell lysates increased after ORFV infection. IL- 8 and decorin reached their peak at almost the same time, but TNF- $\alpha$ was still rising at the time of the last sample collection (Fig. 2). The increase in IL-8 and decorin levels in the cell supernatant is presented in Fig. 3. However, TNF- $\alpha$ was not detected in cell-free supernatants.

IL-8, TNF- $\alpha$, and decorin levels in samples collected at indicated time points after ORFV infection or from controls were detected by cytokine array. Data are expressed as the mean and standard deviation of three replicates.

IL-8 and decorin levels in samples collected at indicated time points after ORFV infection or in controls were detected by cytokine array. The data are expressed as the mean and standard deviation of three replicates. 


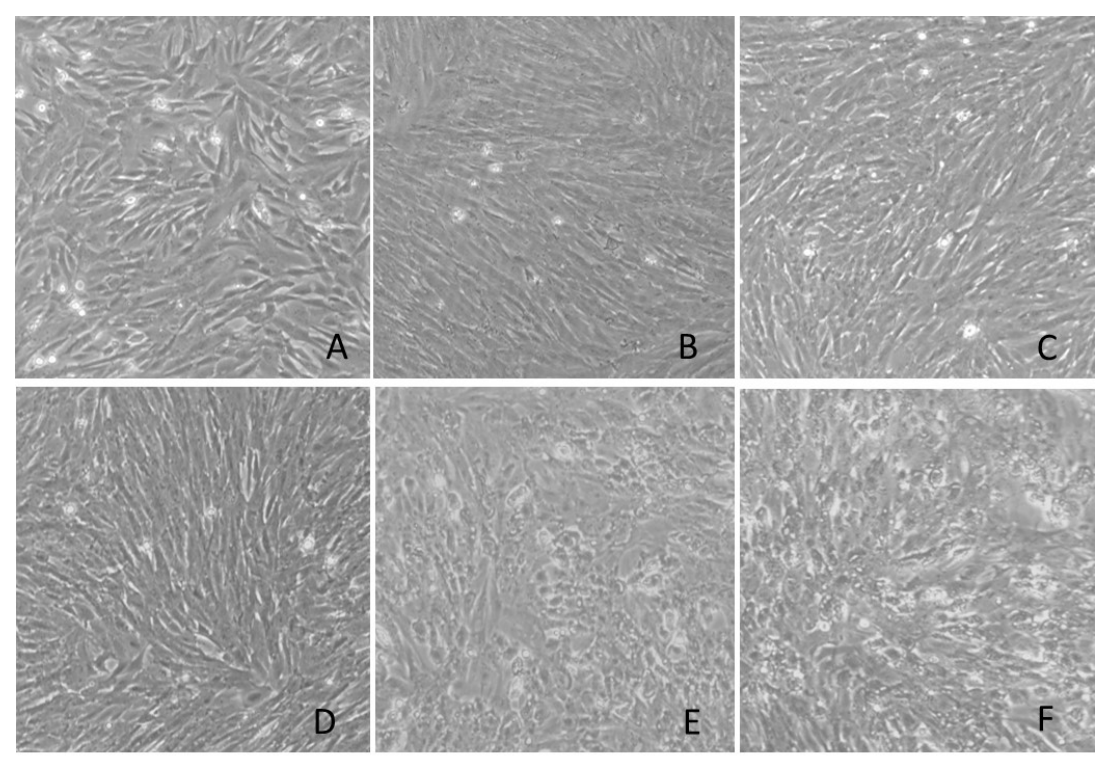

Fig. 1. The GSF cell morphological changes at indicated time points after ORFV infection A $-0.5 \mathrm{~h}, \mathrm{~B}-1 \mathrm{~h}, \mathrm{C}-2 \mathrm{~h}, \mathrm{D}-4 \mathrm{~h}, \mathrm{E}-8 \mathrm{~h}, \mathrm{~F}-12 \mathrm{~h}$
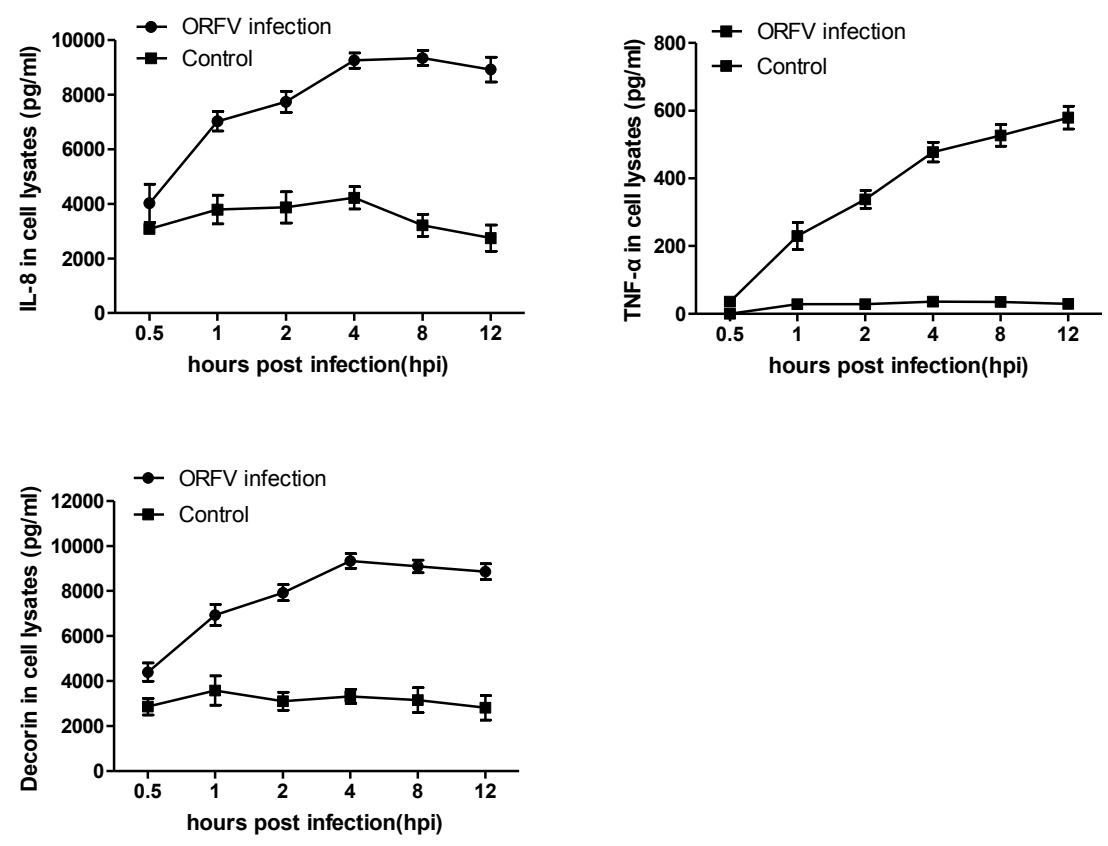

Fig. 2. Changes in interleukin-8 (IL-8), tumour necrosis factor- $\alpha$ (TNF- $\alpha$ ), and decorin levels in cell lysates after ORFV infection
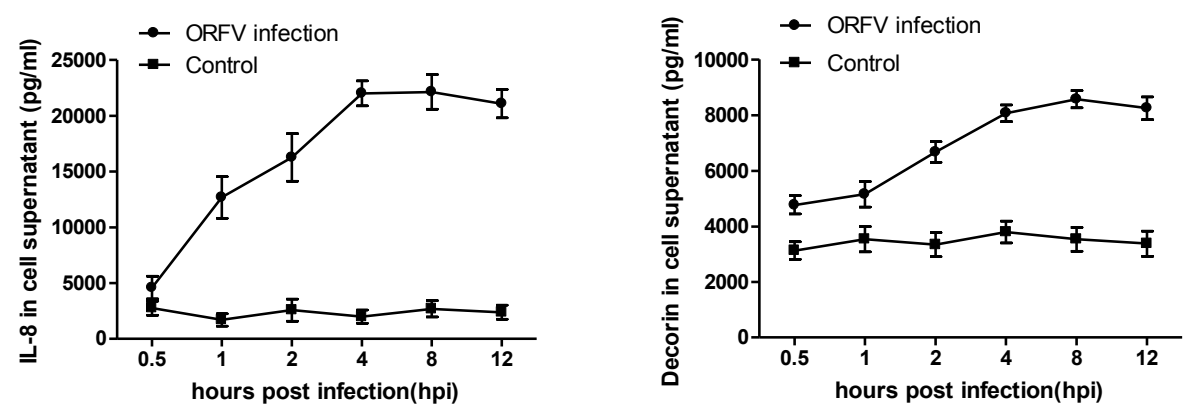

Fig. 3. Changes in interleukin-8 (IL-8) and decorin levels in cell-free supernatant after ORFV infection 


\section{Discussion}

ORFV is an epitheliotropic virus that naturally infects and proliferates in goat and sheep skin. After ORFV infection, dendritic cells, neutrophils, T cells, and $B$ cells accumulate at the infection site (13). ORFV encodes several genes that inhibit the immune response of the host by inhibiting apoptosis, interferon production, inflammatory reaction, and NF-kB signalling $(5,7,12,26)$. Cytokines, which play an important role in the innate immune response after viral infection, might also be affected by the virus. In this paper, we detected differential expression of cytokines in GSF cells after ORFV infection.

Previous studies have indicated that IL- 8 is responsible for neutrophil recruitment (2). In the current study, IL-8 increased both in the cell supernatant and promoted cell lysates after ORFV infection, which is in accordance with a former study which showed that neutrophils are recruited at the ORFV infection site in vivo (1).

The level of TNF- $\alpha$ also increased after ORFV infection, which is in accordance with the results of Anderson et al. (1), who, using an in situ hybridisation method, demonstrated that TNF- $\alpha$ mRNA increased after ORFV infection in vivo. In the cell lysates, the concentration of $\mathrm{TNF}-\alpha$ was on a rising trend during sample collection. TNF- $\alpha$ was detectable only in cell lysates and not in cell-free supernatant, potentially explicable by its concentration in the supernatant not achieving the detection limit.

Decorin was detected in all samples of ORFVinfected cells and in the control but its levels increased with ORFV infection. Decorin has diverse functions, such as regulating extracellular matrix organisation, cell growth, and immune response (24). However, the change in extracellular matrix after virus infection has not been studied so far. Decorin has also been shown to associate with immune cell infiltration and cell cycle arrest in some diseases $(19,28)$. An increase in decorin levels might play a role in recruiting immune cells against virus infection. The concentration of IL-8 and decorin reached their peak at almost the same time after ORFV infection, which may show that both of them were induced at the same stage.

ORFV infection can cause major skin damage either directly by the virus or by the cytokines and chemokines secreted by the host cells. These changes in TNF- $\alpha$, IL- 8 , and decorin might be involved in both the initiation of the antiviral immune response and the pathogenesis of the virus.

In conclusion, this study demonstrated that the early stage of ORFV infection increased IL-8, decorin, and TNF- $\alpha$ levels. This result indicates the response of the target cells of ORFV after their infection. The elevated level of cytokines after infection reported here is a finding which contributes to further studies on the pathogenesis of ORFV and its interaction with the host.
Conflict of Interests Statement: The authors declare that there is no conflict of interests regarding the publication of this article.

Financial Disclosure Statement: This research was supported by the National Natural Science Foundation of China (No.31201914), National Science and Technology Ministry (2015BAD12B04) and National Modern Meat Caprine Industrial Technology System (CARS-39).

Animal Rights Statement: None required.

\section{References}

1. Anderson I.E., Reid H.W., Nettleton P.F., McInnes C.J., Haig D.M.: Detection of cellular cytokine mRNA expression during orf virus infection in sheep: differential interferon-gamma mRNA expression by cells in primary versus reinfection skin lesions. Vet Immunol Immunopathol 2001, 83, 161-176.

2. Das S.T., Rajagopalan L., Guerrero-Plata A., Sai J., Richmond A., Garofalo R.P., Rajarathnam K.: Monomeric and dimeric CXCL8 are both essential for in vivo neutrophil recruitment. PLoS One 2010, 5, e11754.

3. Deane D., McInnes C.J., Percival A., Wood A., Thomson J., Lear A., Gilray J., Fleming S., Mercer A., Haig D.: Orf virus encodes a novel secreted protein inhibitor of granulocytemacrophage colony-stimulating factor and interleukin-2. J Virol 2000, 74, 1313-1320.

4. Delhon G., Tulman E.R., Afonso C.L., Lu Z., de la ConchaBermejillo A., Lehmkuhl H.D., Piccone M.E., Kutish G.F., Rock D.L.: Genomes of the parapoxviruses ORF virus and bovine papular stomatitis virus. J Virol 2004, 78, 168-177.

5. Diel D.G., Luo S., Delhon G., Peng Y., Flores E.F., Rock D.L.: Orf virus ORFV121 encodes a novel inhibitor of NF-kappaB that contributes to virus virulence. J Virol 2011, 85, 2037-2049.

6. Epaulard O., Adam L., Poux C., Zurawski G., Salabert N., Rosenbaum P., Dereuddre-Bosquet N., Zurawski S., Flamar A.L., Oh S., Romain G., Chapon C., Banchereau J., Levy Y., Le Grand R., Martinon F.: Macrophage- and neutrophil-derived TNF-alpha instructs skin langerhans cells to prime antiviral immune responses. J Immunol 2014, 193, 2416-2426.

7. Fleming S.B., Anderson I.E., Thomson J., Deane D.L., McInnes C.J., McCaughan C.A., Mercer A.A., Haig D.M.: Infection with recombinant orf viruses demonstrates that the viral interleukin10 is a virulence factor. J Gen Virol 2007, 88, 1922-1927.

8. Frandsen J., Enslow M., Bowen A.R.: Orf parapoxvirus infection from a cat scratch. Dermatol Online J 2011, 17, 9.

9. Haig D., McInnes C., Deane D., Lear A., Myatt N., Reid H., Rothel J., Seow H.F., Wood P., Lyttle D., Mercer A.: Cytokines and their inhibitors in orf virus infection. Vet Immunol Immunopathol 1996, 54, 261-267.

10. Haig D.M.: Orf virus infection and host immunity. Curr Opin Infect Dis 2006, 19, 127-131.

11. Haig D.M., McInnes C.J.: Immunity and counter-immunity during infection with the parapoxvirus orf virus. Virus Res 2002, 88, 3-16.

12. Haig D.M., McInnes C.J., Thomson J., Wood A., Bunyan K., Mercer A.: The orf virus OV20.0L gene product is involved in interferon resistance and inhibits an interferon-inducible, doublestranded RNA-dependent kinase. Immunology 1998, 93, 335-340.

13. Haig D.M., Thomson J., McInnes C., McCaughan C., Imlach W., Mercer A., Fleming S.: Orf virus immuno-modulation and the host immune response. Vet Immunol Immunopathol 2002, 87, 395-399. 
14. Hosamani M., Scagliarini A., Bhanuprakash V., McInnes C.J., Singh R.K.: Orf: an update on current research and future perspectives. Expert Rev Anti Infect Ther 2009, 7, 879-893.

15. Kuhl J.T., Huerter C.J., Hashish H.: A case of human orf contracted from a deer. Cutis 2003, 71, 288-290.

16. Lateef Z., Fleming S., Halliday G., Faulkner L., Mercer A., Baird M.: Orf virus-encoded interleukin-10 inhibits maturation, antigen presentation and migration of murine dendritic cells. J Gen Virol 2003, 84, 1101-1109.

17. Li A., Dubey S., Varney M.L., Dave B.J., Singh R.K.: IL-8 directly enhanced endothelial cell survival, proliferation, and matrix metalloproteinases production and regulated angiogenesis. J Immunol 2003, 170, 3369-3376.

18. Lin F.Y., Tseng Y.Y., Chan K.W., Kuo S.T., Yang C.H., Wang C.Y., Takasu M., Hsu W.L., Wong M.L.: Suppression of influenza virus infection by the orf virus isolated in Taiwan. J Vet Med Sci 2015, 77, 1055-1062.

19. Mohan H., Krumbholz M., Sharma R., Eisele S., Junker A., Sixt M., Newcombe J., Wekerle H., Hohlfeld R., Lassmann H., Meinl E.: Extracellular matrix in multiple sclerosis lesions: Fibrillar collagens, biglycan and decorin are upregulated and associated with infiltrating immune cells. Brain Pathol 2010, 20, 966-975.

20. Rajkomar V., Hannah M., Coulson I.H., Owen C.M.: A case of human to human transmission of orf between mother and child. Clin Exp Dermatol 2015, 53, 63-65.
21. Reed C.C., Iozzo R.V.: The role of decorin in collagen fibrillogenesis and skin homeostasis. Glycoconj J 2002, 19, 249-255.

22. Rot A., von Andrian U.H.: Chemokines in innate and adaptive host defense: basic chemokinese grammar for immune cells. Annu Rev Immunol 2004, 22, 891-928.

23. Schaefer L., Iozzo R.V.: Biological functions of the small leucine-rich proteoglycans: from genetics to signal transduction. J Biol Chem 2008, 283, 21305-21309.

24. Seidler D.G., Mohamed N.A., Bocian C., Stadtmann A., Hermann S., Schafers K., Schafers M., Iozzo R.V., Zarbock A., Gotte M.: The role for decorin in delayed-type hypersensitivity. J Immunol 2011, 187, 6108-6119.

25. Ware C.F.: The TNF receptor super family in immune regulation. Immunol Rev 2011, 244, 5-8.

26. Westphal D., Ledgerwood E.C., Hibma M.H., Fleming S.B., Whelan E.M., Mercer A.A.: A novel Bcl-2-like inhibitor of apoptosis is encoded by the parapoxvirus ORF virus. J Virol 2007, 81, 7178-7188.

27. Zhang K., Liu.Y., Shang Y., Liu X., Cai X.: Major virulence factors of orf virus and their mechanism for immune evasion. Austin J Infect Dis 2014, 1, 5.

28. Zhao C.X., Zhao Y.T., Wang P.H., Xiao X., Wang D.W.: Influences of recombinant adeno-associated virus-mediated Decorin gene transfection on cell cycle and apoptosis of $\mathrm{SiHa}$ cells. Ai Zheng 2005, 24, 28-32. 